\title{
DESIGN STUDY OF COMPACT CYCLOTRON MAGNET IN VIRTUAL PROTOTYPING ENVIRONMENT *
}

\author{
Bin Qin ", Yongqian Xiong, Yaoyao Xu, Jun Yang, Mingwu Fan \\ Huazhong University of Science and Technology, Wuhan 430074, P. R. China
}

\begin{abstract}
An intelligent magnet design, modelling and optimization method with the aid of beam dynamics analysis and three dimensional magnetic field calculation is introduced. The whole procedure is implemented in an integrated virtual prototyping environment built with python language. As a case study, the main magnet design of a $16 \mathrm{MeV} \mathrm{H}^{-}$compact cyclotron is illustrated. Both the field isochronism and transversal focusing of the beam can be fulfilled, and the mechanical analysis is performed to validate the feasibility in mechanics.
\end{abstract}

\section{INTRODUCTION}

Virtual prototyping (VP), a novel engineering technique, provides a continuous development environment for products. By evaluating virtual prototypes, it can reduce or replace the real physical prototypes, thus saves the cost and time-consuming of products' R\&D. We have applied VP technique to the design and development processes of low-energy cyclotrons, and the framework of cyclotron VP platform (CVPP) has been proposed [1].

The primary goal of CVPP is to provide an integrate environment for multiple design or analysis components covering beam dynamics, magnet, RF cavity, injection line and control system etc. Virtually these modules are heterogeneous. They are written in different languages such as FORTRAN, $\mathrm{C}$ and $\mathrm{C}++$. And some of them are commercial software. How to encapsulate these modules and establish an effective inter-communication method becomes a key issue. The agent based approach with CORBA is a conventional but complicated solution, which requires a long development period. Nowadays, using the high level scripting languages such as Python or Perl becomes an increasingly popular approach to scientific computing [2]. In our solution of CVPP, Python language was used to build the user interface and to be a powerful 'code gluer' for integrating different VP modules [3].

As a crucial task in cyclotron design, main magnet design and optimization includes iterative processes from an initial crude model. The optimized magnetic field distribution should fulfil requirements of isochronisms and transversal focusing of the beam, as well as to avoid dangerous resonance crossing. This paper mainly introduces an automated magnet design, modelling and shimming method under the pythonic integrated

\footnotetext{
*Work supported by National Nature Science Foundation of China: 10435030 .

"bin.qin@mail.hust.edu.cn
}

environment based on virtual prototyping. The detail magnet shimming processes with the support of the 3D magnetic field simulation code TOSCA [4] and an original developed particle tracking code PTP are described.

\section{MAGNET DESIGN AND OPTIMIZATION PROCESSES}

The isochronous average magnetic field of cyclotrons should increase with radius:

$$
B_{\text {iso }}(r)=\gamma(r) \cdot B_{c}=\left(1+T / E_{0}\right) \cdot B_{c}
$$

Where $B_{c}$ is the magnetic field in the center of the cyclotron, $T$ is the kinetic energy of ions, and $E_{0}$ is the rest mass energy.

In low-energy compact cyclotrons, a common method to achieve the isochronous field is to increase the ratio of hill/valley along radius, which called magnet shimming. CVPP takes this method to design the main magnet of cyclotrons.

The magnet optimization is automated in CVPP with the collaboration of magnet design module and beam dynamics analysis module, as shown in Figure 1.

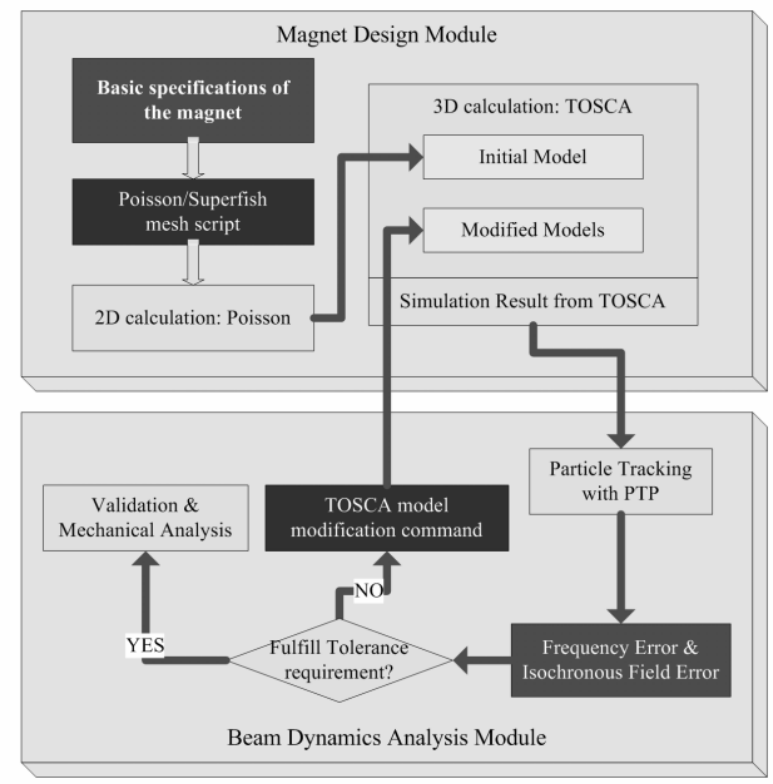

Figure 1. Automated magnet design and optimization process

Based on some basic magnet specifications and 2D calculation result, an initial $3 \mathrm{D}$ magnet model is constructed in OPERA-3D and the magnetic field is 
simulated with TOSCA solver. Then, the magnet design module of CVPP takes some procedures to find an isochronous magnet model from this initial one:

\section{Step 1. Magnetic Field Error Calculation:}

PTP (Particle Tracking Package) is an beam dynamics analysis code written in $\mathrm{C}++$, which can track particles in field map from calculation or measurement. By searching the equilibrium orbits at different particle kinetic energy, it can calculate the frequency error $\Delta f_{\mathrm{p}}(r)$ relate to the design cyclic frequency $f_{\mathrm{p}}$. By using equation (2), the isochronous magnetic field error can be estimated [5].

$$
\Delta B(r)=B_{\text {iso }}(r) \cdot \gamma^{2}(r) \cdot \Delta f_{\mathrm{p}}(r) / f_{\mathrm{p}}
$$

\section{Step 2. Magnet Model Correction:}

The hard-edge magnet model was used to give an approximate solution to convert magnetic field error to corresponding sector angle error, as shown in equation (3).

$$
\Delta \eta(r) \approx \Delta B(r) \cdot \frac{2 \pi / N}{B_{\mathrm{H}}(r)-B_{\mathrm{V}}(r)}
$$

$B_{\mathrm{H}}(r), B_{\mathrm{V}}(r)$ are magnetic field in hill and valley of the magnet pole, $N$ is the sector number of magnet. $\Delta \eta(r)$ is the corresponding sector angle change due to the magnetic field error $\Delta B(r)$. Usually, $\Delta \eta(r)$ used for model correction need to be multiplied with a experience factor $\sigma$ with the value between $0.5 \sim 0.9$ to avoid oscillation of field error during iteration.

The magnet sector angle can be regulated by modifying the control points of the shimming bar attached to the magnet pole. The magnet design module calculates an angle correction list along the radius and generates a model transformation command file for OPERA preprocessor. Thus one complete shimming process is finished. The simulation result of this modified model is checked again in step 1 until the tolerance is achieved.

\section{Step 3. Model Validation and Mechanical analysis:}

When the isochronous magnetic field error is small enough, this validation step starts. PTP will calculate local phase slip to make sure the total phase slip is well controlled. Also, horizontal tune and vertical tune during acceleration is calculated and beam focusing should be satisfied in the magnetic field. If the working point is near the dangerous resonance line or slow resonance crossing happens, the structure of the magnet need to be revised and optimized. Finally, the mechanical analysis including deformation and von Mises stress distribution should be performed.

\section{A CASE STUDY: MAGNET DESIGN OF A $16 \mathrm{MEV} \mathrm{H}^{-}$CYCLOTRON}

A virtual magnet prototype of a $16 \mathrm{MeV} \mathrm{H}^{-}$cyclotron is built for illustration. The main specifications of the magnet are shown in table 1.

Table 1: Magnet Specifications of $16 \mathrm{MeV} \mathrm{H}^{-}$Cyclotron

\begin{tabular}{|l|l|}
\hline Parameters & Value \\
\hline Number of sectors & 4 \\
\hline Sector angle & 50 degree \\
\hline RF frequency & $80.2 \mathrm{MHz}$ \\
\hline Harmonic mode & 4 \\
\hline Average Magnetic Field & $1.35 \mathrm{~T}$ \\
\hline Hill / Valley gap & $0.03 / 0.40 \mathrm{~m}$ \\
\hline Extraction radius & $0.44 \mathrm{~m}$ \\
\hline
\end{tabular}

The initial magnet sector model is shown in fig. 2. One simple straight shimming bar are attached to the side of magnet pole. The shimming bar is parameterized with 20 modifiable edge points.

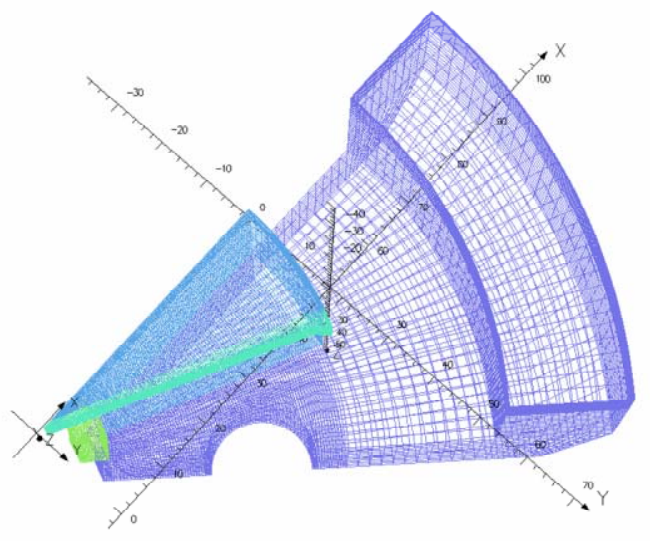

Figure 2. 1/16 parametric magnet model.

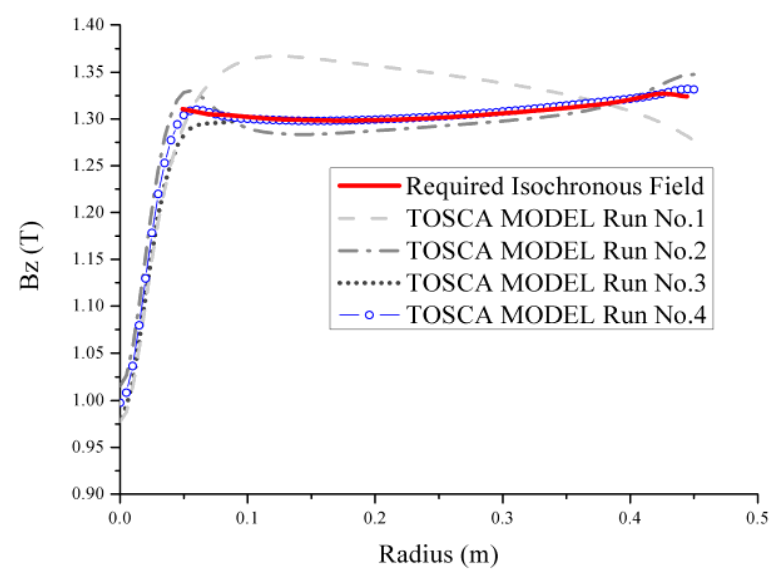

Figure3: Average magnetic field compared with required isochronous field during iterations.

D05 Code Developments and Simulation Techniques 
After 4 automated iterations, the magnetic field satisfies tolerance of isochronisms. The TOSCA simulated average magnetic field during iterations and the required isochronous field is shown in figure 3. Figure 4 shows the final shape of the shimming bar comparing to the initial model. For the optimized model, the orbital frequency error is controlled within $0.1 \%$, and the total phase slip is about 5 degree.
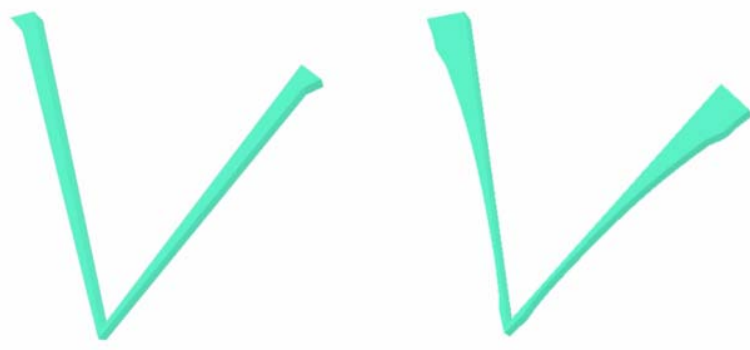

Figure 4: Models of the initial shimming bar and the optimized one after 4 iterations.

Fig. 5 shows the tune diagram during acceleration. As can be observed, both radial and vertical tune are stable. Integer, half integer and Walkinshaw resonance crossing are avoided.

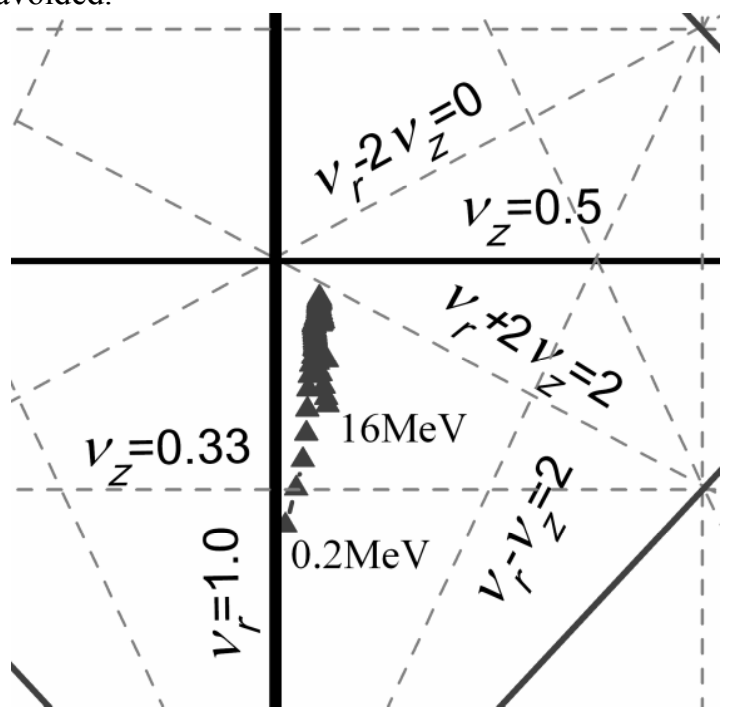

Figure 5: Tune diagram.

The mechanical analysis is important for estimating magnetic field varying due to deformation. The results also can be used to judge whether the von Mises stress exceeds the yielding stress.

Firstly the magnetic force between magnet poles is calculated by TOSCA. In this case, it is $151.6 \mathrm{kN}$ for each sector pair. Mechanical analysis can be performed by taking magnetic, gravitational and vacuum forces as loads. As shown in figure 6, the maximum deformation in pole surface is $65 \mu \mathrm{m}$ at the position of radius $5 \mathrm{~cm}$, with the contribution to the magnetic field error about $0.065 / 15=$ $0.4 \%$. This error can be corrected easily by magnet shimming. Figure 7 shows the maximum von Mises stress is $23 \mathrm{MPa}$ when the cyclotron is working. The stress is far 05 Beam Dynamics and Electromagnetic Fields below the yielding stress, which proves this design is feasible and safety for mechanics consideration.

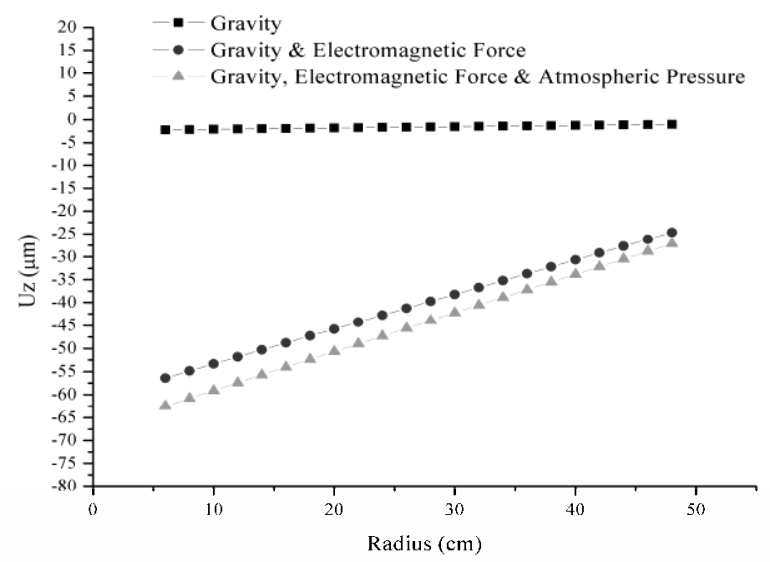

Figure 6: Deformation in the central line of magnet pole surface with different conditions.
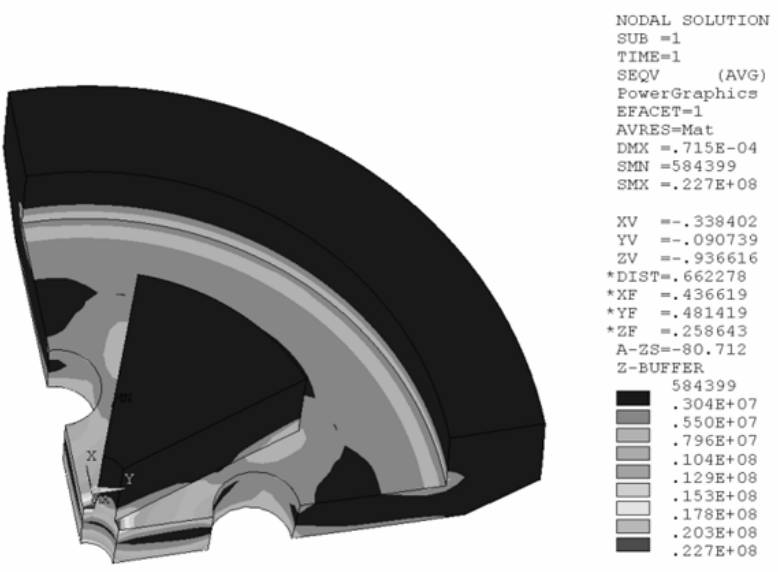

Figure 7: Von Mises stress distribution

\section{CONCLUSION}

This paper described a systematic method for the modeling and correction of the magnet of cyclotrons in the framework of CVPP. The method is validated by virtual magnet models and also can be applied to the magnet measurement and shimming process during construction of cyclotrons.

\section{REFERENCES}

[1] M. Fan et al., "Applying Virtual Prototyping to the Innovative Design of Low Energy Accelerators", PAC 2003, pp. 1560-1562.

[2] D.M. Beazley, "Automated scientific software scripting with SWIG", Future Generation Computer Systems, 19 , 2003, pp. 599-609.

[3] B. Qin et al., "A Pythonic Integrated Solution for Virtual Prototyping of Cyclotrons", NIM B, available online.

[4] “OPERA-3D user guide”, Vector Fields Limited.

[5] Y. Jongen and S. Zaremba, "Cyclotron Magnet Calculations", CERN 96-02, pp. 139-151.

D05 Code Developments and Simulation Techniques 